\title{
Network Architectures for Next Generation Internet Content Distribution
}

\author{
Jianhua He* \\ School of Engineering and Applied Science, Aston University, Birmingham, B4 7ET, United Kingdom
}

The existing Internet architecture has been designed for efficient communication but not for distribution. Since the commercialization of Internet by the 1990s it has achieved huge success and incorporated into virtually every aspect of modern human life. It is estimated that there are about 2.267 billion Internet users on December 31, 2011 [1]. However, with the advances of Internet technologies we are witnessing the reshaping of most traditional communications media including telephone, music, film and television by the Internet. More and more contents are distributed from content providers to many users. It is estimated a high $90 \%$ level of Internet traffic consists of data disseminated to a number of users. With high-quality content such as high definition TV to be disseminated over Internet there will be huge growth of content, which requires scalable and robust network architectures to efficiently distribute over next generation Internet. In this article we analyze several existing network architectures deployed or proposed for future content distribution. The ideas behind these designs will be highlighted and their potentials and issues will be discussed.

In the past decades new network architectures and protocols have been proposed for future Internet. Paul et al. [2] presented a comprehensive survey on the networking research on network architecture for future networks and next generation Internet. They presented a broad perspective of the wide spectrum of research efforts in the following research areas: security, content delivery mechanism, challenged network environments, management and control networks and internetworking layer design [2]. In this article we are focused on content distribution network architectures.

The content distribution mechanisms of the Internet have already evolved from centralized server based distribution mechanisms to distributed approaches of content delivery networks (CDNs). CDN is a network technology which stores copies of data at multiple nodes (especially edge nodes) of a network. It was initially designed to mitigate the load of central servers. The techniques used in the CDN include web caching, server-load balancing, request routing, and content services [3]. A content request may be redirected optimally to a networked cache which could provide service to the request. When strategically designed and implemented a CDN can reduce content access latency and the traffic over the Internet backbones. The content availability and system reliability performances can also be improved due to the distributed and redundant content storage at multiple locations. Most CDNs are operated by application service providers (ASP). But there is an increasing trend of building CDNs by telecommunication service providers (TSP). However it is reported by Leighton [4] that only $50 \%$ of Internet traffic is served from the top 35 core networks, which means there is still a large part of internet traffic could not be provided through CDN. The present state of the art CDNs can only serve cacheable content. Future CDNs must address these challenges to meet the requirements of fast, economic, reliable and scalable content access over next generation Internet.

In contrast to the network based content caching used in CDNs, peer-to-peer networking (P2P), a completely different and disruptive content distribution technology, has been developed over the years [5]. It is a distributed application architecture that divides tasks among equally privileged and equipotent participants in the application. In the $\mathrm{P} 2 \mathrm{P}$ networks peers share their disk storage, processing power and network bandwidth resources. They are both content suppliers and resource consumers, which is in contrast to the traditional client/server model. Major advantages of using P2P networks are its scalability and robustness. The setting up and running costs is very small for the original content distributor. As demand on the content distribution system increases the total capacity of the system also increases. On the other hand, the decentralized nature of P2P networks increases robustness because if a single node in the network fails other nodes with identical content can provide continuous service. As P2P networks do not require a system administrator, the networks have high efficiency and stability. However, the lack of system administrator and the distributed nature of P2P networks introduce new security issues as users may access harmful data. Data availability can be another issue as unpopular files will eventually become unavailable as the users stop sharing them.

As P2P content distribution usually consists of relatively heavy bandwidth usage, it has created problems for Internet Service Providers (ISPs), because P2P traffic can make network saturated and create congestion for other types of Internet use. An approach taken by ISPs to leverage this problem is to implement a form of P2P caching [6]. $\mathrm{P} 2 \mathrm{P}$ caching is similar to content caching used by ISP to accelerate web content. It temporarily store popular P2P content that flows into an ISP's network, therefore network congestion caused by P2P traffic could be reduced and all users could receive improved experiences. It is noted that $\mathrm{P} 2 \mathrm{P}$ caching may improve the network bandwidth usage of $\mathrm{P} 2 \mathrm{P}$ based content distribution in some extent by the means of network caching, although the caching is operated by ISP instead of ASP as done with CDNs. However the problems of security and content associated with $\mathrm{P} 2 \mathrm{P}$ content distribution are not solved with $\mathrm{P} 2 \mathrm{P}$ caching. It is also a challenge for ISP to implement $\mathrm{P} 2 \mathrm{P}$ caching as the $\mathrm{P} 2 \mathrm{P}$ packets need to be identified before they can be cached inside the networks.

Moving a further step beyond content caching, an alternative approach to network architecture for content distribution has been proposed recently, which is called content-centric networking (CCN) or networking named data $[7,8]$. CCN is motivated by the observation that the current Internet use is dominated by content distribution while the network protocols were designed for communications between hosts. It is built on IP's engineering principles but using named content rather than host identifiers as its central abstraction $[7,8]$. In CCN all content is digitally signed and private content is encrypted. It aims to provide simple, scalable, secure, and efficient content distribution services [7]. It is noted that content chunks are stored in the network caches rather than the whole files as with CDNs. However,

${ }^{*}$ Corresponding author: Jianhua He, School of Engineering and Applied Science, Aston University, Birmingham, B4 7ET, United Kingdom, E-mail: j.he7@aston.ac.uk

Received February 27, 2012; Accepted March 01, 2012; Published March 04 2012

Citation: He J (2012) Network Architectures for Next Generation Internet Content Distribution. J Telecommun Syst Manage 1:e104. doi:10.4172/21670919.1000e104

Copyright: (c) $2012 \mathrm{He} \mathrm{J}$. This is an open-access article distributed under the terms of the Creative Commons Attribution License, which permits unrestricted use, distribution, and reproduction in any medium, provided the original author and source are credited. 
the change of communication paradigm from using host identifiers to using named content may pose problems for certain types of network activities such as real-time multimedia applications. This new paradigm of architectural design is also subject to thorough test and validation, which may be extremely difficult to do over current large scale Internet. From an initial systematic evaluation of the suitability of existing software and hardware components in today's routers for the support of CCN, it is reported by Perino and Varvello [9] that a CCN deployment may be feasible at a CDN and ISP scale, but today's technology is not yet ready to support an Internet scale deployment.

In summary there have been extensive research efforts towards content distribution over Internet. We discussed several typical approaches proposed for content distribution over next generation Internet. It can be observed that these proposed approaches could potentially significantly improve the content distribution performances in terms of network bandwidth usage, content access latency and etc. However there are still many open issues such as security, efficiency and network management. There is still an a long way to go in the design and test of scalable, robust, secure and efficient network architecture for next generation Internet content distribution.

\section{References}

1. Internet World Stats (2011)

2. Subharthi Paul, Jianli Pan, Raj Jain (2011) Architectures for the future networks and the next generation Internet: A survey. Computer Communications 34 $2-42$.

3. Hofmann, Markus, Leland R, Beaumont (2005) Content Networking: Architecture, Protocols, and Practice. Morgan Kaufmann Publisher.

4. Leighton T (2008) Improving performance in the Internet. ACM Queue 20-29.

5. Androutsellis-Theotokis S, Spinellis D (2004) A survey of peer-to-peer content distribution technologies. ACM Computing Surveys 36.

6. Bandara HM, Dilum N, Jayasumana AP (2011) Exploiting communities for enhancing lookup performance in structured P2P systems. IEEE Int Conf on Communications (ICC '11).

7. Jacobson V, Smetters DK, Thornton JD, Plass MF, Briggs N, et al. (2009) Networking named content. Proceedings of the 5th ACM International Conference on Emerging Networking Experiments and Technologies (CoNEXT 2009), Rome, Italy, 1-4.

8. Jacobson V, Smetters DK, Thornton JD, Plass MF, Briggs N, et al. (2012) Networking named content. Communications of the ACM 55: 117-124.

9. Diego Perino, Matteo Varvello (2011) A Reality check for content centric networking. ICN'11, August 19, Toronto, Ontario, Canada. 